\title{
Rotational anomalies of mesoscopic rings
}

\author{
R. Merlin \\ Harrison M. Randall Laboratory of Physics, University of Michigan, Ann Arbor, MI 48109-1120, USA
}

Received 19 August 1993; accepted for publication 1 September 1993

Communicated by L.J. Sham

\begin{abstract}
The electronic contribution to the moment of inertia of single-channel metal rings is analyzed. In rings threaded by a magnetic flux, the electron moment $I_{\mathrm{e}}$ exhibits a periodic pattern with maxima at $\Phi_{0}=h c / e$ or $\frac{1}{2} \Phi_{0}$ (modulo $\Phi_{0}$ ) depending on the number of electrons. For slowly rotating rings, $I_{e}$ diverges in the limit of weak disorder. The rotational anomalies rely on the same interference mechanism responsible for persistent currents and the Aharonov-Bohm effect.
\end{abstract}

Electrons in small metallic rings exhibit intriguing coherent phenomena such as persistent currents [110] and the Aharonov-Bohm effect [11-17]. In recent years, the interest in this area has expanded dramatically following the theoretical proposal by Büttiker, Imry and Landauer [1] and major advances on the experimental front, particularly in the field of nanofabrication $[6,15]$. In this Letter, the rotational behavior of a mesoscopic ring is considered. The main results are that the electron component of the moment of inertia depends strongly on magnetic flux and that it diverges in the limit of weak disorder. These anomalies are closely related to other mesoscopic phenomena based on quantum interference [1-17]. Our findings strictly apply to one-dimensional rings at temperature $T=0$ in the absence of inelastic scattering, but we argue that, within some range, real rings should exhibit anomalies as well.

Consider $N$ electrons of mass $m$ confined to a single-channel ring of radius $r$. The system rotates with constant angular velocity $\Omega$ about the axis passing through the center of the ring and normal to its plane. There is a uniform magnetic field $B$ acting on the electrons and a net magnetic flux $\Phi$ through the ring. The one-particle Hamiltonian in the rotating frame, $\hat{H}_{\mathrm{R}}=\hat{H}-\Omega \hat{p}_{\varphi}[18]$, reads

$$
\begin{gathered}
\hat{H}_{\mathrm{R}}=\frac{1}{2 m r^{2}}\left(\hat{p}_{\varphi}+\hbar \nu\right)^{2}-\frac{1}{2} m r^{2} \Omega^{2} \\
-\hbar \Omega\left(\Phi / \Phi_{0}\right)+\hat{V}_{\mathrm{I}}(\varphi)-\gamma \boldsymbol{B} \cdot \hat{\mathrm{s}} .
\end{gathered}
$$

Here, $\hat{H}$ is the Hamiltonian in the inertial frame, $\Phi_{0}=h c / e$ is the flux quantum and $\hat{p}_{\varphi}$ is the angular momentum operator conjugate to the angle $\varphi$ specifying the position of the electron; $\nu=\Phi / \Phi_{0}+$ $m r^{2} \Omega / \hbar, \hat{s}$ is the electron spin, $\gamma$ is the gyromagnetic ratio and $\hat{V}_{\mathrm{I}}(\varphi)$ is the potential due to impurities. For $\hat{V}_{\mathrm{I}}=0$, the eigenfunctions of $\hat{H}_{\mathrm{R}}$ are $\psi_{n}=$ $\exp (\mathrm{i} \varphi) / \sqrt{2 \pi}$ with eigenenergies

$$
\begin{aligned}
\epsilon_{n} & =\frac{\hbar^{2}}{2 m r^{2}}\left[(n+\nu)^{2}+\left(\Phi / \Phi_{0}\right)^{2}-\nu^{2}\right] \\
& \pm \frac{1}{2} \gamma \hbar|\boldsymbol{B}| .
\end{aligned}
$$

The lowest-lying one-particle states for the Aharonov-Bohm configuration $(B=0$, but $\Phi \neq 0)$ are shown in fig. 1. Impurity scattering generally removes the degeneracies at $\nu=0$ and $\frac{1}{2}$ (modulo 1 ) which are analogous to those at $\Phi=0$ and $\frac{1}{2} \Phi_{0}$ (modulo $\Phi_{0}$ ) for $\Omega=0$ [1-4]. We note that the spectrum of fig. 1 is periodic in $\nu$ for stationary $(\nu=\Phi /$ $\Phi_{0}$ ) [1-4], but not for rotating rings, i.e., $\epsilon_{n}(\nu)=$ $\epsilon_{n-1}(\nu+1)$ at $\Omega=0$.

In the following, we consider the limit $\hat{V}_{1 \rightarrow 0}$ and assume that the eigenstates extend throughout the ring ( $2 \pi r$ is smaller than the localization length). As discussed in the context of persistent currents [1-5], one can apply a gauge transformation and concomitant $\nu$-dependent boundary conditions to eliminate $\nu$ from eq. (1) [19]. It is well known that this transformation affects extended but not localized states 


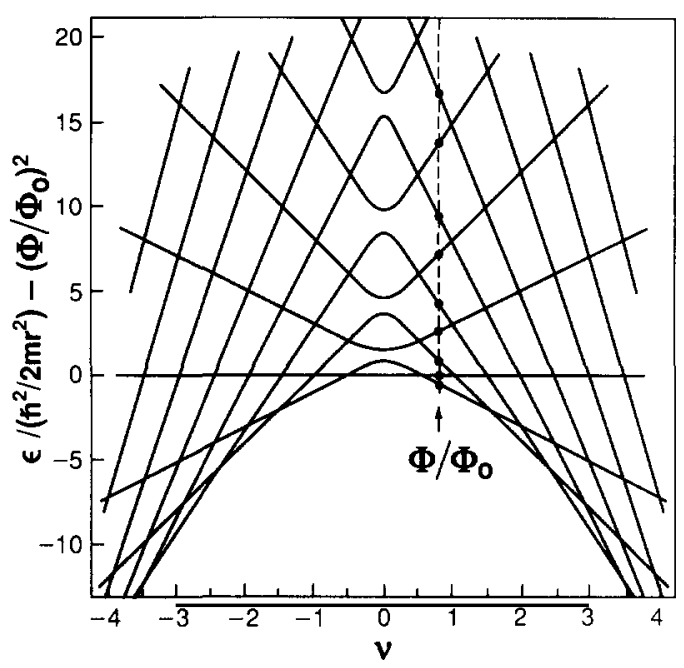

Fig. 1. One-particle energy levels, $\epsilon$, as a function of $\nu$ (rotating frame); $\Phi$ is the magnetic flux. Spin splittings are not included. The moment of inertia is determined by the behavior of states in the vicinity of $\nu=\Phi / \Phi_{0}$, i.e, $\Omega=0$. Coupling to impurities removes degeneracies at $\nu=0, \pm \frac{1}{2}, \pm 1, \pm \frac{3}{2}$, etc. For clarity, this is shown only for $\nu=0$. Dots represent occupied electron states.

[19]. For the latter, the rotational motion leads to a trivial negative shift of the energies by the amount $\frac{1}{2} m r^{2} \Omega^{2}+\hbar \Omega\left(\Phi / \Phi_{0}\right)$.

The results in fig. 1 provide the basis for understanding mechanical anomalies. Let $E_{\mathrm{R}}(\Phi, \Omega)$ be the ground state energy of the non-interacting $N$-electron system. The total angular momentum at $T=0$ is $L=-\partial E_{\mathrm{R}} / \partial \Omega$ [18] and, thus, the electronic component of the moment of inertia is

$I_{\mathrm{e}}(\Phi)=\left(\frac{\partial L}{\partial \Omega}\right)_{\Omega=0}=-\sum_{n}\left(\frac{\partial^{2} \epsilon_{n}}{\partial \Omega^{2}}\right)_{\Omega=0}$.

The sum is for $\epsilon_{n} \leqslant \epsilon_{\mathrm{F}}$ where $\epsilon_{\mathrm{F}}$ is the Fermi energy ( $I_{\mathrm{e}}$ should be distinguished from the differential moment of inertia $\mathscr{I}_{\mathrm{e}}=\partial L / \partial \Omega$ applying to $\Omega \neq 0$ ). Like $E_{\mathrm{R}}$ at $\Omega=0$ [1-5], the moment is a periodic function of $\Phi$ with period $\Phi_{0} . I_{\mathrm{e}}$ is, of course, always positive (the second-order correction to the ground state energy is always negative) and it can be shown to satisfy the sum rule

$\int I_{\mathrm{e}} \mathrm{d} \Phi=m r^{2} N \Phi_{0}$

where the integral is over a period. Not unexpect- edly, the contribution of localized states to $I_{\mathrm{e}}$ is $m r^{2}$ per state irrespective of $\Phi$.

For a sufficiently weak impurity potential, the electrons decouple from the ions $\left(\partial^{2} \epsilon_{n} / \partial \Omega^{2}=\right.$ $\partial^{2} \epsilon_{n} / \partial \nu^{2} \approx 0$ and, thus, $I_{\mathrm{e}} \approx 0$ ) except in the immediate vicinity of $\nu=0$ and $\frac{1}{2}$ (modulo 1 ) where levels mix. At small coupling, these crossings determine the $\hat{V}_{I}$ dependence of the eigenenergies and, therefore, of $I_{\mathrm{e}}$. In particular, the changes of sign of $\partial \epsilon_{n} / \partial \nu$ (at $\nu \approx 0, \pm \frac{1}{2}$, etc.) account for the sum rule requiring that, for some $\Phi, I_{\mathrm{e}} \rightarrow \infty$ as $\hat{V}_{\mathrm{l}} \rightarrow 0$. Parenthetically, we note that the differential moment $\mathscr{I}_{\mathrm{e}}$ exhibits associated divergencies at $m r^{2} \Omega / \hbar= \pm \frac{1}{2}, \pm 1$, etc. for $\Phi=0$. Since terms of eq. (3) due to occupied coupled states (hereafter referred to as pairs) cancel in lowest order, the moment of inertia is determined by those few unpaired electrons at or just below $\epsilon_{F}$. Near crossings, standard degenerate perturbation theory gives

$I_{e}=\frac{1}{4} N^{2} \hbar^{2}\left|V_{I}^{\left(\epsilon_{F}\right)}\right|^{2}\left(\left|V_{I}^{(\epsilon F)}\right|^{2}+\Delta^{2}\right)^{-3 / 2}$.

Here, $\Delta=\delta \phi\left(\hbar^{2} N / 4 m r^{2}\right)$ with $\delta \phi=\Phi / \Phi_{0}-1$ or $\Phi / \Phi_{0}-\frac{1}{2}$ (modulo 1) depending on whether the crossing is at $\Phi=\Phi_{0}$ or $\frac{1}{2} \Phi_{0} .\left|V_{1}^{\left(\epsilon_{\mathrm{F}}\right)}\right|\left(\ll \hbar^{2} N / m r^{2}\right)$ is the matrix element of $\hat{V}_{\mathrm{I}}$ associated with the unpaired states at $\epsilon_{F}$. Reflecting the discontinuity of $\left(\partial \epsilon_{n} / \partial \nu\right)_{\nu=0}$ at $\hat{V}_{\mathrm{I}}=0$ (fig. 1 ), it is clear that $I_{\mathrm{e}}(0)$ becomes arbitrarily large with decreasing impurity scattering. For $\boldsymbol{B}=0$ and $\frac{1}{2} N$ even (odd), the non-zero contributions to $I_{\mathrm{e}}$ originate from two opposite-spin unpaired states diverging at $\Phi \approx 0\left(\frac{1}{2} \Phi_{0}\right)$. If $N$ is odd, the relevant term operating at both $\Phi \approx 0$ and $\frac{1}{2} \Phi_{0}$ is due to a single electron. Assuming that characteristic energies associated with $\hat{V}_{\mathrm{I}}$ are large compared with $\gamma \hbar|\boldsymbol{B}|$, these considerations apply also to cases where $\boldsymbol{B} \neq 0$.

An alternative picture of the rotational anomalies is shown in fig. 2. Here, the inertial-frame ground state energy $E=E_{\mathrm{R}}+\Omega L$ [18] is plotted as a function of $\nu$. In terms of $E, I_{\mathrm{e}}=\left[\partial E / \partial\left(\frac{1}{2} \Omega^{2}\right)\right]_{\Omega=0}$. Reminiscent of a superfluid [20], the steps in the $\hat{V}_{\mathrm{I}}=0$ data correspond to thresholds for which the rotational energy matches particular excitations of the ( $\Omega=0$ ) ground state. Level-mixing due to disorder smooths out the ladder. At $\Phi=0$ and $\frac{1}{2} \Phi_{0}$ (modulo $\Phi_{0}$ ), it also produces the dip associated with the $\hat{V}_{1} \rightarrow 0$ divergency. We notice that, as the states lo- 


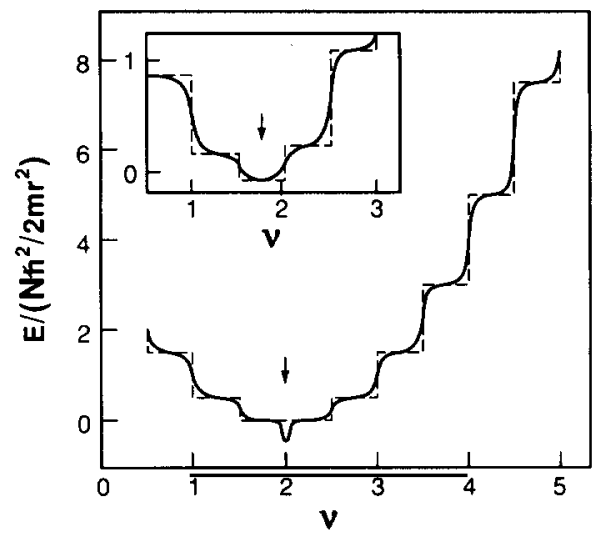

Fig. 2. Ground state energy in the inertial frame, $E$, as a function of $\nu$. Values of $\Phi / \Phi_{0}$ are denoted by arrows. Dashed and full lines correspond, respectively, to $\hat{V}_{\mathrm{I}}=0$ and $\hat{V}_{\mathrm{I}} \neq 0$ (schematic). The main figure, with $\Phi / \Phi_{0}=2$, illustrates the case where coupling of electrons to the impurities is largest and $I_{\mathrm{e}}$ is enhanced. In the inset $\left(\Phi / \Phi_{0}=1.7\right)$, the interaction at $\Omega=0$ is negligible.

calize, the ladder turns into the continuous parabola $E=$ const $+\frac{1}{2} m r^{2} N \Omega^{2}$.

To conclude, we briefly address the problem of a real ring bearing on the experimental significance of our analysis. Although we do not have a definitive answer for this matter, there are compelling arguments suggesting that the $\Phi$ dependence of $I_{\mathrm{e}}$ should fall in an experimentally meaningful range. This is based primarily on the realization that the underlying mechanism of rotational anomalies is very similar to that of persistent currents [1-10]. In particular, the rotational effect also vanishes if the length of the ring is larger than the localization or the phase coherence length [2-5]. Moreover, it is apparent that the moment of inertia $I_{\mathrm{e}}=\partial E / \partial\left(\frac{1}{2} \Omega^{2}\right)$ and the current $j=-\partial E / \partial \Phi$ are but different response functions of the same state. Based on the fact that mesoscopic persistent currents have been found in experiments [6], the analogy suggests that similar rings should exhibit rotational anomalies as well. It remains to be seen whether current processing techniques can successfully overcome the obvious fabrication problems posed by a rotating geometry.

This work was supported by the US Army Research Office under contract no. DAAL-03-92-G0233.

\section{References}

[1] M. Büttiker, Y. Imry and R. Landauer, Phys. Lett. A 96 (1983) 365 .

[2] R. Landauer and M. Büttiker, Phys. Rev. Lett. 54 (1985) 2049.

[3] H.F. Cheung, E.K. Riedel and Y. Gefen, Phys. Rev. Lett. 62 (1989) 587.

[4] G. Montambaux et al., Phys. Rev. B 42 (1990) 7647.

[5] B.L. Altshuler, Y. Gefen and Y. Imry, Phys. Rev. Lett. 66 (1991) 88.

[6] L.P. Lévy et al., Phys. Rev. Lett. 64 (1990) 2074; V. Chandrasekar et al., Phys. Rev. Lett. 67 (1991) 3578.

[7] D. Loss, P. Goldbart and A.V. Balatsky, Phys. Rev. Lett. 65 (1990) 1655.

[8] V. Ambegaokar and U. Eckern, Phys. Rev. Lett. 65 (1990) 381.

[9] D. Loss, Phys. Rev. Lett. 69 (1992) 343.

[10] M. Abraham and R. Berkovits, Phys. Rev. Lett. 70 (1993) 1509.

[11] Y. Aharonov and D. Bohm, Phys. Rev. 115 (1959) 485.

[12] D.Yu. Sharvin and Yu.V. Sharvin, Pis'ma Zh. Eksp. Teor. Fiz. 34 (1981) 285 [JETP Lett. 34 (1981) 272 ].

[13] M. Büttiker et al., Phys. Rev. B 31 (1985) 6207.

[14] A.D. Stone, Phys. Rev. Lett. 54 (1985) 2692.

[15] S. Washburn and R.A. Webb, Adv. Phys. 35 (1986) 375.

[16] N. Byers and C.N. Yang, Phys. Rev. Lett. 7 (1961) 46.

[17] F. Bloch, Phys. Rev. B 2 (1970) 109.

[18] L.D. Landau and E.M. Lifshitz, Statistical physics (Pergamon, Oxford, 1980) p. 104.

[19] D.J. Thouless, in: Physics in one dimension, eds. J. Bernasconi and T. Schneider (Springer, Berlin, 1981) p. 306.

[20] R.E. Packard and T.M. Sanders Jr., Phys. Rev. Lett. 22 (1969) 823;

P. Hakonen and O.V. Lounasmaa, Phys. Today 40 (1987) 70 , and references therein. 